\title{
Atuação do enfermeiro como gestor na rede básica de saúde
}

Um dos mais graves problemas sociais do Brasil é a má qualidade da saúde pública. O objetivo desta pesquisa é ampliar os conhecimentos sobre o gerenciamento da enfermagem quanto o tempo de espera por atendimento nas Unidades Básicas de Saúde. Trata-se de um estudo descritivo de abordagem qualitativa do tipo revisão literária. Foram utilizados artigos e textos científicos indexados nas bases de dados Biblioteca Virtual em Saúde, Biblioteca Eletrônica Científica Online (SCIELO), Literatura Latino-Americana e do Caribe em Ciências da Saúde (LILACS) e BIREME. A insatisfação dos usuários do sistema público de saúde fomenta a necessidade de compreender a dinâmica dos serviços de saúde e na implementação de novos modelos de organização. Foi evidenciado que a atenção primária, necessita de enfermeiros capacitados quanto as suas atribuições gerenciais e organizativas, principalmente quanto ao processo de trabalho para atender a demanda, enfrentando os desafios apontados por este sistema cheio de deficiências.

Palavras-chave: Gerenciamento de Enfermagem; Tempo de espera; Atendimento.

\section{Nurse's performance as manager in the basic health network}

One of the most serious social problems in Brazil is the poor quality of public health. The objective of this research is to expand the knowledge about nursing management regarding the waiting time for care in Basic Health Units. This is a descriptive study with a qualitative approach, such as a literary review. Scientific articles and texts indexed in the Virtual Health Library, Biblioteca Eletrônica Científica Online (SCIELO), Literatura Latino-Americana e do Caribe em Ciências da Saúde (LILACS) e BIREME. The dissatisfaction of users of the public health system promotes the need to understand the dynamics of health services and the implementation of new organizational models. It was evidenced that primary care needs nurses trained in their managerial and organizational duties, mainly in the work process to meet the demand, facing the challenges pointed out by this system full of deficiencies.

Keywords: Nursing Management; Waiting time; Attendance.

Topic: Enfermagem em Saúde Pública

Reviewed anonymously in the process of blind peer.

Kezia dos Santos Araújo

Faculdade Guaraí, Brasil

http://lattes.cnpq.br/8796905785251257

keziaholz@hotmail.com

Lucimar Dias Reis

Faculdade Guaraí, Brasil

lucimardias03@gmail.com

Rogério Carvalho de Figueredo (iD)

Faculdade Guaraí, Brasil

http://lattes.cnpq.br/2845056129867931

http://orcid.org/0000-0003-3349-4812

rogeriocfigueredo@hotmail.com
Received: 15/02/2021

Approved: 22/03/2021

\author{
Reobbe Aguiar Pereira \\ Faculdade Guaraí, Brasil \\ http://lattes.cnpq.br/7447115724350334 \\ enfreobbe@gmail.com
}

\section{Referencing this:}

ARAÚJO, K. S.; REIS, L. D.; FIGUEREDO, R. C.; PEREIRA, R.. Atuação do enfermeiro como gestor na rede básica de saúde. Scire Salutis, v.11, n.2, p.83-92, 2021. DOI: http://doi.org/10.6008/CBPC2236$\underline{9600.2021 .002 .0009}$ 


\section{INTRODUÇÃO}

Sabe-se que a enfermagem é uma profissão que tem evoluído muito nos últimos anos. Nesse novo cenário o enfermeiro está em constantes buscas para aprimorar a qualidade de sua profissão, frente às grandes transformações que estão ocorrendo no mercado de trabalho e especificamente nas unidades de saúde. Assim o profissional de enfermagem precisa estar preparado para o gerenciamento das novas situações que surgem no seu dia-a-dia de trabalho assim como frente às mudanças organizacionais e estruturais dos serviços públicos de saúde.

Especialmente no que se refere às UBS - Unidades Básicas de Saúde as situações de mudanças estão em evidência visto que a população exige mais organização e qualidade daqueles que usam os serviços públicos em saúde.

Em termos de gerenciamento do atendimento nas UBS, há um claro desejo tanto dos usuários como dos profissionais que atuam nessas instituições, de diminuir o tempo espera por atendimento. Isso requer reformulações burocráticas e (ou) técnicas, sendo uma questão de organização do trabalho e compreensão das normas que conduzem a agilidade no atendimento, sem deixar, claro, de respeitar as normas de segurança e priorizar a qualidade do atendimento oferecido (BRASIL, 2012).

Por outro lado, ainda se busca compreender até em que ponto os entraves para a promoção da agilidade do atendimento nas UBS estão na esfera da política de saúde pública, decorrentes da burocratização do processo. A priori, tem-se o pensamento de que para melhorar a qualidade dos serviços e atendimentos realizados nas unidades de saúde, além do gerenciamento de enfermagem é necessária vontade política daqueles que dirigem a saúde, especialmente na esfera de gestão pública (BRASIL, 2012).

Um importante fator a ser gerenciado é o tempo de espera nessas unidades, que tem sido alvo de um grande número de reclamações pelos usuários do sistema público de saúde. Diante do exposto, surge a seguinte problemática: $O$ que o enfermeiro da atenção primária pode fazer para diminuir o tempo de espera por atendimento?

Portanto, acredita-se que diante da importância e da necessidade de reflexão sobre a temática, levando em consideração a escassez de literatura disponível, justifica-se a elaboração deste artigo. Acredita-se que o gerenciamento de enfermagem pode se destacar em alguns pontos no sentido de minimizar os entraves burocráticos, realizando um atendimento mais individualizado e mais humanizado, selecionando os casos que realmente necessitam de atendimento prioritário. Estas são estratégias que podem contribuir para o melhoramento do atendimento dentro das UBS.

Com o presente trabalho espera-se contribuir com os profissionais de enfermagem, especialmente na compreensão das necessidades humanas que devem ser levadas seriamente em consideração quando o indivíduo procura ajuda na esfera da saúde pública. Assim sendo, fazer um estudo sobre as políticas gerenciais da saúde, especialmente na área da enfermagem, é de grande relevância para os que acreditam numa gestão mais eficaz da enfermagem.

É evidente que um trabalho dessa natureza amplia também significativamente os conhecimentos do pesquisador sobre a relação que o Estado tem com o serviço de saúde, mostrando que muitas vezes o 
elemento político interfere negativamente naquilo que se pretende fazer para melhorar a qualidade dos serviços de enfermagem, especialmente na questão da melhoria do atendimento nas UBS.

A gerência, portanto, deve ser compreendida como atribuição dos dirigentes, profissionais e pacientes na perspectiva de construção de um projeto que atenda às necessidades da sociedade e que esteja voltado para a integralidade num processo cotidiano como proposta de mudança. Assim, o gerente atua como interlocutor e mediador do processo de trabalho.

Diante do exposto com este trabalho objetiva-se em âmbito geral ampliar os conhecimentos sobre o gerenciamento da enfermagem quanto o tempo de espera por atendimento nas UBS.

Em âmbito mais específico o estudo objetiva: a) Identificar os principais fatores que interferem no tempo de espera por atendimento nas Unidades Básicas de Saúde; b) Evidenciar a importância do gerenciamento de enfermagem na organização do atendimento; c) Descrever e apontar estratégias que possam contribuir para redução do tempo de espera por atendimento nas UBS.

\section{METODOLOGIA}

Este estudo constituiu-se de uma revisão de artigos com base em uma pesquisa bibliográfico, descritivo e qualitativo. A coleta dos dados para fundamentação dessa pesquisa se que unas bases de dados da Biblioteca Virtual em Saúde (BVS), LILACS, BIREME e Google Acadêmico, usando os descritores: Gerenciamento dos Serviços de Saúde, Atendimento na Atenção Primária à Saúde, Tempo de espera no SUS, Organização do atendimento em saúde, Gerenciamento de Enfermagem. Como critérios de inclusão para seleção do material foram utilizados artigos completos em língua portuguesa, referentes a pesquisas de campo e bibliográfica, publicadas do ano de 2012 a 2018.

\section{DISCUSSÃO TEÓRICA}

\section{A insatisfação dos usuários do SUS quanto ao atendimento prestado e quais suas consequências}

A literatura que se refere à satisfação ou à insatisfação do povo brasileiro com relação ao acolhimento e (ou) à qualidade do atendimento, especialmente nas UBS tem evidenciado que mudanças na concepção de saúde e de qualidade de vida, ou seja, para a maioria dos usuários dos serviços públicos de saúde não basta que se faça um bom acolhimento, o atendimento e diversos outros aspectos também são levados em consideração, especialmente quanto à abrangência desse atendimento (AVELINO, et al., 2015).

Os noticiários nacionais e internacionais vez por outra destacam o descrédito do povo brasileiro com relação à qualidade dos serviços em termos de saúde, especialmente no que ser refere ao acolhimento/atendimento, ressaltando ser de má qualidade. No entanto, essa crítica poucas vezes se refere ao quantitativo de UBS, ou seja, se observa um substancial crescimento do número de UBS (ALMEIDA et al., 2017).

Esse crescimento, quando se analisa na forma simples quantitativa é real, mas não implica, necessariamente, na alteração das tradicionais formas de atenção à saúde ou na estratégia de promoção de equidade. Geralmente os reclamantes se referem à ocorrência de baixa cobertura e um processo de 
trabalho ainda pautado na abordagem curativo-reparadora (CAVALCANTI, et al., 2012).

Mas tudo é conjuntural, se os gestores da saúde pública, bem como os profissionais assistencialistas se esforçarem, com certeza haverá qualidade no atendimento e os usuários estarão mais satisfeitos. Tem-se ressaltado que as instituições devem ser espaços de produção, para prestação de serviços de qualidade aos usuários, mas também deve haver a valorização do potencial criativo dos sujeitos que trabalham nessas instituições: gestores, trabalhadores e usuários (CIAMPONE et al., 2014)

As UBS devem ser a porta de entrada para todos os serviços disponíveis em saúde pública. São elas as instituições que congregam o atendimento inicial dos usuários do SUS. A atenção primária em saúde que o Estado deve dar para os usuários, no que diz respeito à qualidade e agilidade do atendimento deve estar voltada para as UBS. É na atenção primária que se organiza e se proporciona a otimização de recursos, tanto básicos, como especializados e que se mantém o vínculo e a responsabilização pelas necessidades de saúde das pessoas, das famílias e da comunidade. Mas isso não tem acontecido de forma satisfatória e os motivos são diversos (MARIN et al. 2013).

É oportuno destacar aqui os principais motivos do alongamento do tempo e espera por atendimento nas UBS, na visão da maioria dos usuários. O que abaixo se expõe é resultante de uma pesquisa feita por estudiosos que investigam a questão da demora por atendimento nas UBS (MARIN et al., 2013).

As pesquisas realizadas por esses estudiosos chegaram aos seguintes resultados: Falta de políticas organizacionais específicas para o atendimento nas UBS. Quando as políticas são pensadas e postas em práticas levam em consideração o sistema como um todo, mas poderia ver casos mais específicos. Quando se vai construir uma UBS não se leva em consideração a facilidade de acesso do usuário. É visto que outros fatores são preponderantes à facilidade do acesso do usuário. O número de postos é insuficiente com relação à demanda. A população brasileira cresceu bastante e a construção dos postos de saúde não acompanhou esse crescimento. O número de profissionais, na maioria dos casos é insuficiente para atender à demanda. A disponibilidade de horário para o atendimento, geralmente é incompatível com as necessidades dos usuários (eles têm a necessidade de chegarem muito cedo à unidade para conseguir o agendamento e, mesmo assim, encontram dificuldades). Há um inexplicável alongamento do período para a realização da consulta, que às vezes pode demorar até meses. Há também um excesso de burocracia no preenchimento dos formulários para o agendamento das consultas. As vagas para consulta com médicos especialistas, geralmente são muito restritas e demandam bastante tempo, pois o número desses profissionais é insuficiente na rede pública de saúde (NAKAMURA, 2014).

Em termos mais voltados para o gerenciamento do quadro funcional se destaca a necessidade de oferta de serviços contínuos, oportunos, que atendam à demanda real e que sejam capazes de assegurar acesso aos demais níveis de atenção primária. É importante ressaltar que ainda há a questão do ingresso no serviço público por meios de apadrinhamento político, desrespeitando a via do concurso público. O não respeito aos horários estabelecidos pela instituição (há médicos que chegam a atrasar até duas horas). Falta ao serviço sem o prévio aviso à administração da UBS. Falta de fiscalização e punição para os 
infratores (MARIN et al., 2013).

Como se pode ver são muitos as questões apontadas como negativos e por isso, devem ser prioridades no gerenciamento de enfermagem na atuação nas UBS visando minimizar o tempo de atendimento (MARIN at al., 2013).

Diante do exposto fica claro que para que o sistema de saúde tenha maior eficiência e eficácia na consecução dos seus objetivos é necessário que as unidades de atendimento primário e acolhimento funcionem seguindo alguns pontos da estratégia da empresa privada, ou seja, adote uma abordagem empresarial, resgatando o pressuposto de racionalidade, segundo o qual os arranjos estruturais corretos reduzem os problemas e ampliam a desempenho. É uma diretriz organizativa que busca benefícios para o sistema público de a saúde. Sabe-se que são muitos desafios, mas é preciso ampliar a eficácia da atenção primária, acabando ou minimizando a heterogeneidade do serviço e as dificuldades de fluxo pelos níveis de atenção (QUINELLATO, 2013).

Não é do desconhecimento de nenhum profissional de saúde que a prolongação do tempo de atendimento, seja em hospitais ou nas UBS se torna potencial risco de agravamento do estado de saúde do cliente. Nesse aspecto a ação do enfermeiro é substancial para que isso não ocorra (SILVA et al., 2018).

O que se pode observar é que há também um significativo descompasso entre à oferta de serviços e as reais necessidades do usuário. Permanecer esperando por algo que não lhe pode ser ofertado é uma grande perda de tempo para o usuário, assim, o enfermeiro deve se antecipar e procurar saber qual tipo de atendimento ao cliente está buscando. Caso não haja a oferta na UBS, ele deve indicar imediatamente outro local que possa atender às necessidades do cliente (NAKAMURA, 2014).

\section{A importância da organização e participação coletiva no cenário da atenção primária em saúde}

Nesse cenário o ideal seria que a atenção primária tenha a reciprocidade através da escuta interessada e a qualidade no processo de trabalho dos profissionais, especialmente os enfermeiros, na formação de vínculo e respeito, sempre com resposta positiva e disposição profissional para a resolução do problema daqueles que procuram os serviços básicos de saúde na esfera pública. Entretanto há ocorrência de troca de sentimentos e atitudes, gestos simbólicos que podem se manifestar de forma positiva e negativa (GARUZI, et al., 2017).

Tem havido no acolhimento consideráveis avanços, no sentido de sair das relações de assistencialismo burocrático, objetivando incorporação do sistema de atendimento da atenção primária, proporcionando o bem-estar físico, mental, social e cultural do usuário, mostrando dedicação mais humana dos profissionais em relação ao atendimento, Isso muitas vezes até pode minimizar os efeitos deletérios (maléficos, prejudiciais) oriundos de instalações e espaço físicos construídos aquém dos padrões de qualidade (GARUZI, et al., 2017).

Portanto, o esforço que deve ser feito cotidianamente e por todos os envolvidos, inclusive pelos usuários do sistema público de saúde. É impossível que o atendimento de qualidade dependa exclusivamente dos trabalhadores em saúde. Assim, deve haver acordos e pactos realizados com o gestor e 
com os companheiros de trabalho, em função das necessidades dos usuários, e ainda se faz necessário que haja modos de incentivos para que os servidores possam se doar mais e se sentirem valorizados (QUINELLATO, 2013).

A participação das pessoas envolvidas em vários níveis de decisão contribui para aumentar a qualidade das decisões e gestão, satisfação e motivação pessoas, bem como a competitividade das organizações. É claro que a colaboração dos gestores nesse processo não é limitada àqueles em posições hierarquicamente formais envolvendo determinadas características, como sistemas de informação, treinamento, recompensas, delegação de autoridade, estilo liderança e cultura organizacional (COSTA, et al., 2011).

O processo de colaboração se estende a todos os membros da organização, sem distinção. O princípio básico da gestão de enfermagem nas unidades de saúde é valorizar e respeitar o usuário como um ser humano. Ele deve ser considerado como um instrumento essencial para a realização de atividades, não meramente como um estorvo que vem tirar a tranquilidade do profissional de saúde (COSTA, et al., 2011).

Neste contexto, compreende-se que as formas de gerenciamento do acolhimento/atendimento feito com qualidade ocorrem a partir da conscientização de todos os envolvidos que o ser humano, apesar de ser individual, é composto por duas dimensões: corpo e espírito, ou seja, a dimensão fisiológica e a psicológica, podendo-se ainda levar em conta algumas características sociais que não sejam de caráter discriminatório (MONTEZELI et al., 2012).

Tal conscientização não ocorrerá espontaneamente, só poderá ocorrer plenamente quando há capacitação dos atuais profissionais de saúde neste sentido. Só a vontade de acolher e atender bem são importantes, mas não é suficiente para desencadear um processo mais amplo dessa nova visão sobre o acolhimento/atendimento nas unidades de saúde (KOPF et al., 2015).

\section{Estratégias que podem contribuir para redução do tempo de espera por atendimento nas UBS}

Uma vez conscientes que as Unidades Básicas de Saúde são a primeira opção de acesso aos serviços de saúde, o profissional de enfermagem deve entender que depende muito de sua ação a satisfação ou não do usuário com o sistema. Assim, ele deve fazer o máximo para se aproximar das expectativas do paciente, do seu desejo de ser bem atendido e de forma que demande menos tempo de espera (KOPF et al., 2015).

Não restam dúvidas que repousa na questão do acolhimento e da qualidade do atendimento a principal diretriz da Política Nacional de Humanização (PNH) que, alguns municípios passaram a implantar na busca de adesão de uma assistência qualificada utilizando o modelo em defesa do direito à vida (WALDOW, 2013).

Nesse cenário, se faz necessário que muitas vezes o enfermeiro saia da sua rotina de trabalho e precise utilizar outras estratégias, ainda que contradigam a proposta de atenção à saúde vigente. A superação da dificuldade do paciente/usuário é muito mais importante para sua carreira profissional de que uma eventual reclamação das instâncias superiores (SILVA et al. 2015).

Esse aspecto parece indicar que somente a ampliação do acesso à porta de entrada não é suficiente 
para resolver os problemas de saúde da população, demandando novas formas de organização dos fluxos de serviços. Talvez seja em consequência dessa condição que, principalmente os usuários da UBS, pontuam como dificuldade a falta de médico especialista na unidade (ALMEIDA et al., 2017).

Antes de mostrar algumas sugestões especificas para melhorar a qualidade do trabalho de atendimento nas UBS, convém discutir em âmbito geral o que é o gerenciamento participativo em termos de enfermagem. É que o entendimento para este estudo passa pela união de forças, não podendo o bom gerenciamento de enfermagem ocorrer de forma isolada (BERNARDES, et al., 2013).

Tradicionalmente as unidades de saúde eram geridas de forma centralizada onde o diretor detinha todos os poderes decisórios, mas estabeleceu-se progressivamente uma significativa discussão, que almeja transformar a gestão da saúde em um instrumento democrático e participativo, oportunizando a toda equipe de saúde e comunidade participarem dos destinos do sistema de saúde e a tomarem parte no processo decisório (AZEVEDO, 2013).

Dessa forma percebe-se que são grandes as preocupações e os esforços investidos em mudanças no gerenciamento do sistema de saúde brasileiro nas últimas décadas, principalmente no final dos anos 80 , do século passado (BERNARDES, et al., 2013).

Acredita-se então que a gestão da saúde quando gerida dentro do espírito democrático é fator de desenvolvimento para a nação, pois, está comprovado que um sistema de saúde bem democrático é a mola de impulso desenvolvimentista das nações, assim como os enfermeiros, são profissionais importantes para a sociedade (AZEVEDO, 2013).

Então, gestão participativa na saúde pública tem que ser transformada em todos os seus aspectos, uma vez que, os enfermeiros não podem mais revestir seu trabalho com sua individualidade, pois a moderna visão democrática de sistema de saúde funciona a partir da existência de uma equipe de trabalho, onde as funções são bem específicas e que todas as decisões são tomadas em conjunto, isto é, a gerência de enfermagem, nos moldes que ditam a modernidade é participativa (BERNARDES, et al., 2013).

A antiga forma de gestão, onde cada profissional tomava suas decisões quase que individualmente, apenas obedecendo aos ditames documentais das instruções dos órgãos superiores da administração de saúde pública ou privada, não pode mais existir e a gestão está sendo profundamente modificada (BERNARDES, et al., 2013).

A equipe de enfermagem deve promover eventos de conscientização de toda a equipe da UBS, a fim de conscientizar a respeito da necessidade de agilidade no atendimento e que isso depende muito do empenho da equipe de atendimento e de servidores em geral. Realizar momentos de educação permanente em saúde, para conscientização, alinhamento e fortalecimento do trabalho em equipe. Colocar em prática as políticas e protocolos, bem como regimentos, instrutivos e modelos de organização preconizados pelo sistema de saúde. Evitar retornos desnecessários à UBS, adiantando a questão dos exames feitos por especialistas. Organizar a dinâmica do atendimento na UBS, e também dos serviços ofertados, criando fluxos ou informativos para os usuários. Agilizar a referência e contra referência dos atendimentos, bem como fortalecer a comunicação com demais unidades de saúde e serviços. Buscar 
selecionar o atendimento pela questão da urgência. Dispor de atendimento integral e multiprofissional. Responsabilizar os profissionais quanto as suas atribuições e conduta no serviço de saúde (CEM, 2015).

Tomando-se essas providências fica evidente que o gerenciamento de enfermagem está sendo bem feito e consegue evitar atrasos no trabalho, faltas injustificadas, triagem dos atendimentos para que se evite desperdício de tempo por parte da equipe e do usuário, entre outras providências (CEM, 2015).

O gerenciamento, quando feito dentro dos parâmetros da responsabilidade social dão bons resultados e a unidade de saúde em pouco tempo apresenta uma significativa evolução na questão da qualidade do atendimento (CEM, 2015).

Quando se analisa o que acima foi exposto percebe-se facilmente que através do gerenciamento de enfermagem é possível melhorar a qualidade do atendimento, especialmente na questão da diminuição da espera por atendimento (DIAS, et al., 2016).

É uma questão de despertamento da consciência crítica dos enfermeiros acerca de sua atuação na gerência das redes básicas de saúde buscando um espaço para desenvolver seu potencial e conquistar o prestígio digno a profissão (DIAS, et al., 2016).

É possível quando o enfermeiro e toda a equipe responsável pelo atendimento/acolhimento tem o compromisso com a sociedade a fim de firmar o papel de cuidador e prestar uma melhor assistência à população. As ideias para melhor gerenciar o trabalho não devem ser miraculosas, mas sim práticas com base nas condições locais e no perfil dos usuários (BRASIL, 2012).

As mudanças só ocorrem quando as ações são bem pensadas e bem situadas, com novas perspectivas para a enfermagem, que busque modificações nas políticas de saúde e no gerenciamento (AZEVEDO, 2013).

Nesse sentido, há uma necessidade implícita na profissão de enfermeiro, quando pretende fazer um bom gerenciamento, que requer abordar os aspectos éticos na ação gerencial. Ainda se vislumbra a adoção desses profissionais no sentido de trabalhar de forma organizada, em que a boa prática decorre da vontade de bem agir. Essa vontade é baseada na internalização de regras aceitas como legítimas. Nessa visão, o aprendizado ocorre em um contexto científico, através de uma educação permanente (TREVIZAN, et al., 2012).

Portanto que a função gerencial do enfermeiro deve ser baseada em valores da profissão, em seu código de ética e nos direitos do paciente, integrando cuidado humano qualificado, guiado pelo respeito, por livre consentimento e pela promoção do paciente como um protagonista e sujeito do cuidado (TREVIZAN, et al., 2012).

Ainda é oportuno reiterar que a gerência de enfermagem tem regras claras e constam nas Diretrizes Curriculares Nacionais como uma ferramenta indispensável que o auxilia no seu cotidiano e nas expectativas do mercado de trabalho, no que tange à qualidade do atendimento, principalmente dentro da perspectiva de consolidação do SUS (WEIRICH, et al., 2013).

Destaca-se ainda que dentre as habilidades gerenciais deva figurar o gerenciamento de recursos humanos que se caracteriza como uma atividade importante para a viabilização das práticas para 
administrar o trabalho das pessoas. Faz parte dessas habilidades, a capacidade de encontrar soluções para a diminuição do tempo de espera por atendimento das pessoas que procuram as UBS (BEZERRA, 2012).

Sabe-se que isso é um desafio no cotidiano de trabalho do enfermeiro, mas não deve lhe faltar disposição e nem confiança para enfrentar e vencer esse desafio. Ao escolher essa profissão deve-se ter a compreensão de que ela é mesmo cheia de desafios, mas não há nada na esfera do impossível (SOUZA et al., 2012).

\section{CONCLUSÕES}

Após análise de tudo o que aqui se escreveu chega-se à conclusão de que os objetivos traçados foram conseguidos, uma vez que se evidenciou que o sistema de saúde brasileiro, especialmente na atenção primária, necessita de enfermeiros capacitados para atender a demanda, enfrentando os desafios apontados por este sistema cheio de falhas.

Ao longo da investigação se descobriu que as dificuldades apresentadas na questão da qualidade do atendimento nas UBS não estão unicamente ligas à ingerência da enfermagem, o problema é bem mais amplo e possui intercorrências geradas em fatores sociais, políticos e econômicos.

Esclareceu-se que a boa gestão da saúde depende da união dos seus diversos setores, especialmente o de enfermagem que para essa ser praticada com qualidade deve ter um bom gerenciamento e que a luta não pode ser desmembrada de outras lutas na questão da qualidade da saúde brasileira, especialmente em âmbito público.

\section{REFERÊNCIAS}

ALMEIDA, M. M.; COUTINHO, L. S.; SANTOS, M. S. Enfermeiro como gerenciador do acolhimento na atenção primária: revisão integrativa. Caxias: FACEMA, 2017.

AVELINO, C. C. V.. Qualidade da atenção primária à saúde: uma análise segundo a intervenção evitáveis em um município de minas gerais-Brasil. Ciência \& Saúde Coletiva, v.20, n.4, p.1285-1293, 2015.

AZEVEDO, S. C.. O Processo de Gerenciamento x Gestão no trabalho do Enfermeiro. Dissertação (Mestrado em Enfermagem) - Universidade do Rio Grande do Norte, Natal, 2013.

BERNARDES, A.; CECÍLIO, L. C. O.; NAKAO, J. R. S.; ÉVORA, Y. D. M.. Os ruídos encontrados na construção de um modelo democrático e participativo de gestão hospitalar. Ciênc. Saúde Coletiva, v.12, n.12, p.74-82, 2013.

BEZERRA, A. L. Q.. O contexto da educação continuada enfermagem na visão dos gerentes de enfermagem e enfermeiros de educação continuada. Monografia (Especialização em Enfermagem) - Universidade de São Paulo, São Paulo, 2012.

BRASIL. Saúde da família: uma estratégia para a reorientação do modelo assistencial. Brasília: Ministério da Saúde, 2012.

BRASIL. SUS: a estrutura e funcionamento das unidades públicas de saúde no Brasil: Ministério da Saúde, 2012.

CAVALCANTI, Y. W.. Avaliação de Usuários da Atenção Básica sobre a Implantação de Protocolo de Assistência Integral Odontológica. Pesquisa Brasileira de Odontopediatria Clínica Integrada, v.12, n.3, p.405-412, 2012.

CEM. Centro de Especialidades Médicas dobra o número de atendimentos e diminui o tempo de espera. PSJP, 2015.

CIAMPONE, M. H. T.; KURCGANT, P.. O Ensino de Administração Hospitalar no Brasil: o processo de construção de competências gerenciais. Revista Brasileira de Enfermagem, v.57, n.4, p.401-7, 2014

COSTA, A.. Enfermeiras e o Paradoxo das Relações SaberPoder no Programa de Controle de Infecções Hospitalares. Tese (Doutorado em Enfermagem) - Universidade Federal do Rio de Janeiro, Rio de Janeiro, 2012.

DIAS, C. L. C; COELHO, C. F.; FONSECA, G. G. P.; PARCIANELLO, M.. Enfermeiro como Gerenciador da Rede Básica de Saúde. Texto Contexto Enferm., v.18, n.2, p.24957,2016

GARUZI, M.; ACHITTI, C. O. M.; SATO, C. A.; ROCHA, A. S.; SPAGNUOLO, R. S.. Acolhimento na estratégia saúde da família: Revisão integrativa. Revista Panamericana de Saúde Pública, v.35, n.2, p.144-149, 2017.

KOPF, Á. W.; HORTALE, V. A.. Contribuição dos sistemas de 
gestão de Carlos Matus para uma gestão comunicativa. Revista Ciência e Saúde Coletiva, v.10, n.4, p.374-382, 2015

MARIN, M. J. S.; MARCHIOLI, M.; MORACVICK, M. Y. A. D.. Fortalezas e fragilidades do atendimento nas unidades básicas de saúde tradicionais e da estratégia de saúde da família pela ótica dos usuários. Texto Contexto de Enfermagem, v.22, n.3, p.780-788, 2013

MONTEZELI, J. H.; PERES, A. M.. Competência Gerencial do Enfermeiro: conhecimento publicado em periódicos brasileiros. Cogitare Enfermagem, v.14, n.3, p.553-558, 2012.

NAKAMURA, F. C.. O princípio do atendimento integral na rede pública de saúde. São Paulo: Faculdade de Direito de Franca, 2014

QUINELLATO, L. V.. A diretriz de hierarquização do SUS: mudando a antiga perspectiva do modelo médicoassistencial privatista. Fundação Getúlio Vargas, v.32, n.5, p. 186-194, 2013.
SILVA, F. H.; BARROS, M. E. B.; MARTINS, C. P..

Experimentação e refeição sobre o apoio institucional em saúde: trabalho a partir da humaniza SUS. Comunicação Saúde, v.8, n.2, p.108-115, 2015

SILVA, P. M.; BARRO, K. P.; TORRES, H. C.. Acolhimento com classificação de risco na Atenção Primária: percepção dos profissionais de enfermagem. Revista Mineira de Enfermagem, v.16, n.2, p.225-231, 2018.

SOUZA, F. A.; PAIANO, M.. Desafios e dificuldades enfrentadas pelos profissionais de enfermagem em início de carreira. UEM, v.15, n.2, p.267-273, 2012.

TREVIZAN, M. A.. Aspectos Éticos na Ação Gerencial do Enfermeiro. Rev. Latino-Am. Enfermagem, v.10, n.1, 2012.

WALDOW, V. R.. Cuidado Humano: o resgate necessário. 10 ed. Porto Alegre: Sagra Luzzatto, 2013.

WEIRICH, C. F.. O Trabalho Gerencial do Enfermeiro na Rede Básica de Saúde. Texto contexto-enfermagem, v.18, n.2, p. 528-535.

A CBPC - Companhia Brasileira de Produção Científica (CNPJ: 11.221.422/0001-03) detém os direitos materiais desta publicação. Os direitos referem-se à publicação do trabalho em qualquer parte do mundo, incluindo os direitos às renovações, expansões e disseminações da contribuição, bem como outros direitos subsidiários. Todos os trabalhos publicados eletronicamente poderão posteriormente ser publicados em coletâneas impressas sob coordenação da Sustenere Publishing, da Companhia Brasileira de Produção Científica e seus parceiros autorizados. Os (as) autores (as) preservam os direitos autorais, mas não têm permissão para a publicação da contribuição em outro meio, impresso ou digital, em português ou em tradução. 